\title{
Glutamatergic mechanisms associated with stress-induced amygdala excitability and anxiety-related behavior
}

\author{
Sophie Masneuf ${ }^{\mathrm{a}, 1}$, Emily Lowery-Gionta ${ }^{\mathrm{b}, 1}$, Giovanni Colacicco ${ }^{\mathrm{a}}$, Kristen E. Pleil ${ }^{\mathrm{b}}$, Chia \\ $\mathbf{L i}^{\mathrm{b}}$, Nicole Crowley ${ }^{\mathrm{b}}$, Shaun Flynn ${ }^{\mathrm{a}}$, Andrew Holmes ${ }^{\mathrm{a}}$, and Thomas Kash ${ }^{\mathrm{b},{ }^{*}}$ \\ aLaboratory of Behavioral and Genomic Neuroscience, National Institute on Alcoholism and \\ Alcohol Abuse, NIH, Bethesda, MD, USA \\ bLaboratory of Molecular Neurophysiology, Bowles Center for Alcohol Studies, Department of \\ Pharmacology, School of Medicine, University of North Carolina at Chapel Hill, NC, USA
}

\section{Abstract}

The neural factors underlying individual differences in susceptibility to chronic stress remain poorly understood. Preclinical studies demonstrate that mouse strains vary greatly in anxietyrelated responses to chronic stress in a manner paralleled by differential stress-induced changes in glutamatergic signaling in the basolateral amygdala (BLA). Previous work has also shown that alterations in the amygdala gene expression of the GluN1 NMDA and the GluK1 kainate receptors are associated with stress-induced alterations in anxiety-like behavior in the C57BL/6J mouse strain. Using in vivo behavioral pharmacological and ex vivo physiological approaches, the aim of the current study was to further elucidate changes in glutamate neurotransmission in the BLA caused by stress and to test the functional roles of GluN1 and GluK1 in mediating stress-related changes in behavior. Results showed that stress-induced alterations in anxiety-like behavior (light/ dark exploration test) were absent following bilateral infusion of the GluK1 agonist ATPA into the BLA. Intra-BLA infusion of the competitive NMDA antagonist AP5 produced a generalized behavioral disinhibition/locomotor hyperactivity, irrespective of stress. Slice electrophysiological recordings showed that ATPA augmented BLA GABAergic neurotransmission and that stress increased the amplitude of network-dependent spontaneous excitatory postsynaptic currents and amplitude of GABAergic miniature inhibitory postsynaptic currents in BLA. These findings could indicate stress-induced BLA glutamatergic neuronal network hyperexcitability and a compensatory increase in GABAergic neurotransmission, suggesting that GluK1 agonism augmented GABAergic inhibition to prevent behavioral sequelae of stress. Current data could have implications for developing novel therapeutic approaches, including GluK1 agonists, for stress-related anxiety disorders.

\section{Keywords}

Stress; Basolateral amygdala; GluN1; GluK1; Glutamate; C57BL/6J

() 2014 Elsevier Ltd. All rights reserved.

*Corresponding author. thomas_kash@med.unc.edu, thomas.kash@gmail.com, tkash@email.unc.edu (T. Kash).

${ }^{1}$ Joint first authorship. 


\section{Introduction}

Psychological stress is a major risk factor for various neuropsychiatric conditions and individuals differ in their sensitivity to stress, partly due to predisposing genetic and neurobiological factors (Caspi et al., 2010). Rodent models offer an experimentally tractable strategy for studying the effects of stress on behavior, and then delineating the mediating neural factors (Holmes and Singewald, 2013). We have previously found that following stress C57BL/6J mice showed a pattern ostensibly indicative of decreased anxiety-like behavior (i.e., more time in the light compartment of the light/dark exploration test and open arms of the elevated plus-maze). However, further analysis suggests that this stress-induced response in C57BL/6J mice can be interpreted as an anxiety-related drive to escape the apparatus or an 'active-coping' response (Mozhui et al., 2010; Uchida et al., 2011) - a hypothesis supported by the finding that the response was effectively reversed by chronic treatment with the anxiolytic fluoxetine (Ihne et al., 2012).

The neural and molecular basis of these stress-induced behavioral abnormalities currently remains unclear. The basolateral amygdala (BLA) is activated by stressors (Singewald, 2007; Whittle et al., 2010) and is a major target of chronic stress (Duvarci and Pare, 2007; Kavushansky and Richter-Levin, 2006; Maroun et al., 2013; Reznikov et al., 2009; Roozendaal et al., 2009). Gene expression analysis of multiple corticolimbic regions, including the BLA, from C57BL/6J before and after chronic stress exposure revealed differential expression of multiple genes (Mozhui et al., 2010). Intriguingly, there was increased gene expression of Grinl (GluN1 subunit NMDA receptor) and decreased expression of Grik1 mRNA (GluK1, formerly GluR5, kainate receptor subtype) in the BLA of C57BL/6J mice following stress.

These prior findings are of particular interest given GluN1 and GluK1 are important regulators of amygdala synaptic transmission and plasticity (Aroniadou-Anderjaska et al., 2012; Lack et al., 2008; Li and Rogawski, 1998; Wu et al., 2007), and more generally, in the context of a growing literature implicating amygdala glutamate signaling in stress-related anxiety (Cryan and Dev, 2007; Griebel and Holmes, 2013; Krishnan et al., 2007; Surget et al., 2009). The functional roles of amygdala GluK1 and GluN1 in mediating stress-induced anxiety in C57BL/6J mice are unclear, as is the precise nature of the stress-induced changes in amygdala glutamate-mediated synaptic transmission. The major aim of the current study was to address these questions using a combination of in vivo behavioral pharmacological and ex vivo electrophysiological approaches.

\section{Material and methods}

\subsection{Subjects}

Subjects were 2-3 month old male C57BL/6J mice obtained from The Jackson Laboratory (Bar Harbor, ME, USA). Following shipping, mice were acclimated to the vivarium for at least 1 week prior to experimental manipulation in a temperature- $\left(22 \pm 3{ }^{\circ} \mathrm{C}\right)$ and humidity(45 $\pm 15 \%$ ) controlled vivarium under a 12-h light/dark cycle (lights on at 06:00). Food and water were provided ad libitum. Separate cohorts of mice were used for the behavioral and electrophysiological experiments. All experimental procedures were approved by the 
NIAAA and UNC Animal Care and Use Committees, and followed the National Institutes of Health guidelines outlined in "Using Animals in Intramural Research."

\subsection{Implantation of amygdala-targeting guide cannula}

Mice were anesthetized with isoflurane and bilaterally implanted via stereotaxic surgery (Kopf Instruments, Tujunga, CA, USA) with 26-gauge indwelling cannulae (Plastics One, Roanoke, VA, USA) targeting the BLA, as previously described (Gunduz-Cinar et al., 2013). The coordinates were AP $-1.4, \mathrm{ML} \pm 3.3, \mathrm{DV}-3.8$ to -4.1 . Mice were single-housed following surgery and given a 1-week recovery period before stress during which dummy cannulae were intermittently manipulated to habituate mice to handling and prevent cannula blocking.

To verify cannula placements at the completion of testing (see Fig. 1B), mice were perfused with $4 \%$ paraformaldehyde. Fixed brains were sectioned (50 $\mu \mathrm{m}$ thickness) on a vibrating microtome (American Optical 860, San Marcos, USA) and stained with cresyl violet to aid localization of cannula tips. Cases in which cannula were not bilaterally localized in the BLA were excluded. To further confirm the localization of infusion, some mice were infused with a fluorophore (BODIPY ${ }^{\circledR}$ TMR-X, SE, 5 mM in PBS, DMSO 40\%, Invitrogen, Eugene, OR, USA) and brain (50 $\mu$ m-thick) sections visualized with a SteREO Lumar V12, Fluorescent Microscope (Carl Zeiss Microscopy LLC, Thornwood, USA) equipped with a CY3 filter (545/25 nm excitation, 565 lpxr dichroic, and 605/70 nm emission) (see Fig. 1C).

\subsection{Repeated restraint stress}

Mice were placed in ventilated $50 \mathrm{~mL}$ Falcon tubes for $2 \mathrm{~h}$ per day (10:00-12:00 h) for 10 consecutive days, while non-restrained mice remained in their home cage, as previously described (Ihne et al., 2012; Mozhui et al., 2010). Body weight was measured before and after the 10-day stress period to confirm that stress led to a loss of body weight (Ihne et al., 2012; Mozhui et al., 2010).

\subsection{Drug infusion and behavioral testing}

One day after the final stress exposure, mice were tested on the light/dark exploration test (LDE) for anxiety-related behavior (Crawley, 1981; Yang et al., 2008) (for schematic of experimental design, see Fig. 1A). The apparatus and procedure was as previously described (Ihne et al., 2012; Mozhui et al., 2010). The apparatus was an opaque black Plexiglas shelter $(39 \times 13 \times 16 \mathrm{~cm})$ with a $13 \times 8 \mathrm{~cm}$ aperture at floor level that opened onto a large white Plexiglas square arena $(39 \times 39 \times 35 \mathrm{~cm})$ illuminated to $~ 90$ lux. Mice were placed halfway, head-forward, in the shelter to begin a 15-min session. The latency to first exit the shelter and enter the light compartment, the number of entries into the light compartment, the accumulated time spent in the light compartment, and the accumulated distance traveled in the light and dark compartments during the first 5 min of the session was measured by the Ethovision videotracking system (Noldus Information Technology Inc., Leesburg, VA, USA).

Mice were randomly assigned to 1 of 3 drug groups: $2.5 \mu \mathrm{g}$ AP5 sodium salt (NMDAR antagonist), 125 pmol ATPA (GluK1 agonist), or $0.9 \%$ saline vehicle. Drug concentrations 
were chosen based on previous studies in rats or mice (Holmes et al., 2012; Lack et al., 2008; Walker et al., 2002). Fifteen minutes prior to testing, bilateral 33-gauge injectors (Plastics One, Roanoke, VA, USA) with $1 \mathrm{~mm}$ projection were lowered into the indwelling guide cannulae. A volume of $0.3 \mu \mathrm{l}$ per hemisphere was infused via a syringe pump (Harvard Apparatus PHD 22/2000, Holliston, MA, USA) at a rate of $0.25 \mu \mathrm{l} \mathrm{per} \mathrm{minute.} \mathrm{To}$ ensure diffusion into the brain and minimize reflux, custom-modified injector needles with a $1.2-1.3 \mathrm{~mm}$ projection beyond the cannula tip were inserted prior to infusion to produce a small space in the tissue, and injectors were left in place for $5 \mathrm{~min}$ to allow for complete diffusion and then removed. Mice remained in the homecage until testing.

\subsection{Slice electrophysiology}

One day following the final exposure to stress, mice were sacrificed via deep isoflurane anesthesia and decapitation, and brain slices containing the BLA were prepared as previously described (Li et al., 2012; Mozhui et al., 2010). Briefly, following sacrifice, brains were rapidly removed and $300 \mu \mathrm{m}$ slices were cut on a Leica VT1000S vibratome (Leica Biosystems, Buffalo Grove, IL, USA) in a cold $\left(1-4{ }^{\circ} \mathrm{C}\right)$, sucrose-based external solution. Slices were then immediately placed in normal ACSF at $30^{\circ} \mathrm{C}$ and allowed to recover for at least $1 \mathrm{~h}$. Following recovery, individual slices were placed in a holding chamber and continuously perfused with normal ACSF maintained at $30^{\circ} \mathrm{C}$ at a rate of 2 $\mathrm{mL}$ per minute. Neurons were visualized using infrared video microscopy (Olympus, Center Valley, PA, USA). Recording electrodes (3-5 M $\Omega$ ) were pulled with a Flaming-Brown Micropipette Puller (Sutter Instruments, Novato, CA, USA), using thin-walled borosilicate glass capillaries. Signals were acquired by a Multiclamp 700 B amplifier (Molecular Devices, Sunnyvale, CA, USA), digitized at $10 \mathrm{kHz}$, and analyzed using Clampfit 10.2 software (Molecular Devices, Sunnyvale, CA, USA). Input resistance and access resistance were continuously monitored throughout all experiments, and those in which changes in access resistance exceeded $20 \%$ were excluded from all data analyses.

Whole-cell voltage clamp recordings were performed to test for alterations in synaptic plasticity, as measured by AMPAR/NMDAR ratio, a putative marker for synaptic potentiation (Humeau et al., 2007), using a cesium gluconate-based internal solution [(in mM) 117 gluconic acid, 20 HEPES, 0.4 EGTA, 5 TEA, $2 \mathrm{MgCL}_{2}$, 2 QX-314, $4 \mathrm{Na}_{2} \mathrm{ATP}$, $0.4 \mathrm{Na}_{2} \mathrm{GTP}, \mathrm{pH}$ 7.3, 285-290 mOsmol]. Evoked NMDAR-mediated synaptic transmission was examined by placing a stimulating electrode in the dorsal part of the BLA and then measuring the relationship between stimulus intensity and peak amplitude of evoked NMDAR mediated currents. Fast synaptic transmission, which is primarily AMPARmediated (Christian et al., 2013), was assessed from recording pharmacologically isolated spontaneous synaptic events at a membrane potential of $-70 \mathrm{mV}$, in the presence of picrotoxin.

To simultaneously record both spontaneous excitatory and inhibitory events from individual neurons, the recording configuration was altered by adjusting the intracellular solution, and then recording at the reversal potential for the opposing current, as previously described for other brain regions (Kim et al., 2013). Specifically, inhibitory postsynaptic currents (IPSCs) were recorded at $+10 \mathrm{mV}$, the reversal potential for AMPAR-mediated currents, and 
excitatory postsynaptic currents (EPSCs) were recorded at $-55 \mathrm{mV}$, the reversal potential for GABAR-mediated events, using a cesium methanesulfonate-based intracellular solution [(in $\mathrm{mM}) 135$ cesium methanesulfonate, $10 \mathrm{KCl}, 1 \mathrm{MgCl}_{2}$, 0.2 EGTA, 2 QX-314, 4 MgATP, 0.3 GTP, 20 phosphocreatine, $\mathrm{pH} 7.3,285-290 \mathrm{mOsmol})$. To determine network-independent synaptic transmission, recordings were repeated in the presence of the sodium channel blocker tetrodotoxin, which blocks action potential firing and thereby recurrent networkdriven transmission.

GluK1 signaling in the BLA has been suggested to effect anxiety-like behavior by modulating the excitability of GABAergic interneurons, based on the observation that intraBLA injection of the GluK1 blockade increases anxiety-like behavior while application of the GluK1 agonist, ATPA, depolarizes interneurons and increases spontaneous GABAergic transmission in this region (Wu et al., 2007). To test for alterations in GluK1 modulation of BLA function, recordings were made after pharmacologically isolating GABAergic transmission by including $50 \mu \mathrm{M}$ APV (NMDAR antagonist) and $100 \mu \mathrm{M}$ GYKI53655 (AMPAR antagonist) in the extracellular solution. IPSCs were recorded at $+10 \mathrm{mV}$ using a cesium gluconate-based internal solution. Following the establishment of a stable baseline, the GluK1 agonist, ATPA, was bath applied at a concentration of $3 \mu \mathrm{M}$ for $10 \mathrm{~min}$ followed by a 10 min washout period.

\subsection{Statistical analysis}

The effect of stress exposure and drug treatment on body weight and behavioral measures was analyzed using 2-factor analysis of variance (ANOVA). Bonferonni-adjusted post-hoc tests were used to probe significant ANOVA terms. As effects of stress and drug treatment were predicted a priori, planned t-tests were conducted to compare groups in the absence of significant ANOVA terms. Outliers were identified as cases with behavioral scores $>2$ standard deviations from each overall group mean, resulting in the exclusion of one case from each of the following groups: non-stressed/vehicle, non-stressed/AP5, stressed/vehicle, non-stressed/ATPA. For electrophysiology experiments, the effects of stress on synaptic plasticity were assessed using $t$-tests (using Welch's correction where variances were unequal). The effects of stress on the amplitude of NMDAR currents across a range of stimulation intensities were analyzed using 2 -factor (treatment $\times$ stimulus intensity) ANOVA, with repeated measures for stimulus intensity. The effects of stress on the response to bath application of ATPA were normalized to baseline values and assessed as the percent change from baseline using 2 -factor (time $\times$ treatment) ANOVA, with repeated measures for time. The threshold for statistical significance was set at $P<.05$.

\section{Results}

\subsection{Effects of intra-BLA AP5 and ATPA on stress-induced LDE test behavior}

There was a significant effect of stress on pre- versus post-stress body weight, due to a significant decrease in body weight in all 3 groups of stressed mice relative to a significant increase in non-stressed controls over the same period (effect of stress: F1,37 $=98.95, P<$. 01) (Fig. 2A). 
In the LDE test, there was a significant main effect of stress on percent light time $(\mathrm{F} 1,37=$ $7.20, P<.05)$, light entries $(\mathrm{F} 1,37=4.25, P<.05)$ and distance traveled in the light $(\mathrm{F} 1,37=$ $9.00, P<.01)$, as well as a significant main effect of drug treatment on percent light time $(\mathrm{F} 2,37=4.44, P<.05)$, light entries $(\mathrm{F} 2,37=16.20, P<.01)$ and distance traveled in the light compartment $(\mathrm{F} 2,37=17.97, P<.01)$, but no significant stress $\times$ treatment interactions.

Bonferroni-adjusted post-hoc comparisons probing the main effect of drug demonstrate that AP5 treatment significantly increased percent light time $(P<.05)$, light entries $(P<.05)$ and distance traveled in the light compartment $(P<.05)$, relative to both vehicle treatment and ATPA treatment, irrespective of stress. Bonferonni-adjusted post-hoc comparisons probing the main effect of stress demonstrate that stress did not significantly alter LDE behaviors in vehicle- or ATPA-treated mice, but did significantly increase percent light time $(P<.05)$ and distance traveled in the light compartment $(P<.05)$, but not entries in to the light $(P>$. 05), in AP5 treated mice relative to AP5 treated controls. Because previous data from our lab has demonstrated that $\mathrm{C} 57 \mathrm{BL} / 6 \mathrm{~J}$ mice spend more time in the light compartment following a restraint stress protocol identical to the one used in the current study, we performed planned $t$-test comparisons to probe the effects of drug treatment on stressinduced LDE behavior (Mozhui et al., 2010; Uchida et al., 2011). These comparisons showed that vehicle-treated stressed mice spent significantly more percent time in the light $(t(11)=2.53, P<.05)$ and traveled farther in the light (but not dark) compartment $(t(11)=$ $2.29, P<.05$ for light; $t(11)=0.88, P>.05$ for dark, data not shown), and showed a trend for more light entries $(t(11)=2.16, P=.053)$, as compared to vehicle-treated non-stressed controls (Fig. 2B-D). Thus, in agreement with previous reports, stress increased preference for the light compartment. Notably, stress-related effects on percent time in the light were absent in mice infused with ATPA $(t(14)=0.25, P>.05$ for percent time in light; $t(14)=$ $0.27, P>.05$ for entries in light; $t(14)=0.52, P>.05$ for distance traveled in light), suggesting that activation of BLA kainite receptors attenuates the effects of stress. In mice infused with AP5, stress-related effects were not observed in entries in light $(t(12)=1.505$, $P>.05)$, though stress-related effects were apparent in distance traveled in the light $(t(12)=$ $2.220, P<.05)$ and a non-significant trend was observed for percent time in light $(t(12)=$ $2.006, P>$.05). Thus, AP5 treatment appeared to augment the effects of stress on specific aspects of LDE behavior. Because potential non-specific locomotor effects of the drugs could confound the effects of stress on LDE behavior, total distance traveled during the entire 15-min assay was analyzed (Fig. 2D inset). There were significant main effects of stress $(\mathrm{F} 1,37=6.594, P<.05)$ and $\operatorname{drug}(\mathrm{F} 2,37=34.39, P<.05)$ on total distance traveled for the duration of the 15-min assay. Bonferonni-adjusted post-hoc comparisons revealed the AP5 significantly enhanced the total distance traveled as compared to both the vehicle treatment and ATPA treatment, regardless of stress exposure $(P<.05)$. In addition, stress significantly enhanced total distance traveled only in AP5-treated mice $(P<.05)$. Thus, AP5 treatment non-specifically enhanced locomotion. Further analysis with planned comparison $t$-tests to probe specific drug and stress effects on locomotion demonstrates that stress did not alter drug effects on total distance traveled $(t(11)=1.716, P>.05$ for vehicle-treated mice; $t(14)=0.2334, P>.05$ for ATPA-treated mice; $t(12)=2.021, P>.05$ for AP5-treated mice), though a non-significant trend was observed in AP5-treated mice. Thus, while stress did not alter total locomotion in vehicle- and ATPA-treated mice, it enhanced locomotion in AP5-treated mice. 


\subsection{Effects of stress on BLA synaptic transmission}

There was no significant effect of stress on AMPAR/NMDAR ratio (Fig. 3A), NMDARmediated EPSC peak amplitude (effect of current: F9,135 = 55.36, $P<.01$ ) (Fig. 3B), or (primarily) AMPAR-mediated spontaneous EPSC amplitude (Fig. 3D) in BLA pyramidal neurons, suggesting that stress does not alter these aspects of excitatory transmission in the BLA. However, an increase in excitatory trans-mission was evident in the presence of the $\mathrm{GABA}_{\mathrm{A}}-\mathrm{R}$ antagonist picrotoxin, as a significant increase in the frequency of spontaneous EPSCs was observed in stressed mice, as compared to non-stressed controls (Welchcorrected $t$-test: $t(11)=2.25, P<.05$ ) (Fig. $3 \mathrm{E}$ ). Thus, in the absence of inhibitory modulation, excitatory transmission in the BLA is enhanced following stress.

We next used an internal solution that allowed for simultaneous recording of inhibitory and excitatory transmission from individual neurons. There was no significant effect of stress on either glutamatergic spontaneous EPSC amplitude or frequency (Fig. 4A-C) or GABAergic spontaneous IPSC amplitude or frequency (Fig. 4D-F). Stress had no significant effect on glutamatergic miniature EPSC amplitude or frequency (Fig. 4F-I), but did significantly increase GABAergic miniature IPSC amplitude, but not frequency (Fig. 4K- L). Thus, stress enhanced local inhibitory modulation in the BLA.

ATPA significantly increased GABAergic spontaneous IPSC amplitude, regardless of stress (ATPA effect: $\mathrm{F} 11,121=11.63, P<.01$, stress effects: ns, ATPA $\times$ stress interaction: ns) (Fig. S1A, B). ATPA also significantly increased GABAergic spontaneous IPSC frequency, irrespective of stress (ATPA effect: F11,121 $=3.57, P<.01$, stress effects: ns, ATPA $\times$ stress interaction: ns) (Fig. S1A, C). Thus, activation of kainite receptors by ATPA enhances BLA GABA transmission, an effect which is not modified by stress.

\section{Discussion}

The results of the current study provide a number of novel insights into the effects of chronic stress on anxiety-like behavior and associated changes in amygdala glutamatergic signaling.

Replicating previous findings (Ihne et al., 2012; Mozhui et al., 2010), C57BL/6J mice chronically exposed to restraint stress showed a significant increase in exploration of the aversive light compartment in the LDE test. This effect is usually indicative of a decrease in anxiety-like behavior (Griebel and Holmes, 2013). However, there are also previous examples in which increased exploration of the aversive areas of anxiety tests, such as the elevated plus-maze, appear to more related to a drive to escape the apparatus than a low level of anxiety (Holmes et al., 2000; Nagura et al., 2012). Moreover, C57BL/6J mice retained certain classic responses to chronic stress, including hypothalamic-pituitary-adrenal (HPA) axis hyperactivity and a robust loss of body weight (current results and Mozhui et al., 2010). Thus, as previously argued (Ihne et al., 2012; Mozhui et al., 2010), this behavior may instead reflect an atypically expressed increased anxiety-like response related to an activecoping or escape-driven response. 
Regardless of the precise nature of the behavioral response to stress, the current study found that the stress-induced increase in light compartment exploration was absent following infusion of the GluK1 kainate receptor agonist, ATPA, directly into the BLA prior to LDE testing. One possibility is that ATPA exerted an anxiolytic-like effect under non-stressed conditions that prevented detection of any further increases due to stress. However, infusion of the drug produced no significant effects in non-stressed mice, suggesting that GluK1 activation may have relieved the effect of stress without affecting anxiety-like behavior under basal conditions. While further studies would be needed to clarify the stressmodifying effects of ATPA, GluK1 is an interesting potential target for such modifiers for a number of reasons.

Previous gene expression analysis found that amygdala GluK1 mRNA expression was significantly reduced in the BLA of C57BL/6J mice as a result of chronic stress exposure (Mozhui et al., 2010). Moreover, GluK1 receptors are well positioned to modulate amygdala responses to stress. The receptor is densely located on the somatodendritic sites of interneurons in the BLA and bidirectionally regulate GABA release, such that activation of GluK1 promotes GABA release (Braga et al., 2003; Li et al., 2001). A previous report found that application of ATPA to BLA-containing brain slices activated GABAergic interneurons and reduced pyramidal neuron excitability, and also that intra-BLA delivery of the GluK1 antagonist LY3882884 increased anxiety-like behavior (Wu et al., 2007). The GluK1mediated increase in GABAergic neurotransmission in the BLA was confirmed in the current study using slice recordings of spontaneous inhibitory postsynaptic currents in the presence of ATPA (although there was no indication that this effect was altered by stress, more detailed analysis would be required to fully exclude such a change). Thus, these findings raise the possibility that ATPA infusion may work to augment GABAergic inhibition and attenuate the excitability of glutamatergic BLA neurons to prevent the behavioral sequelae of stress.

Electrophysiological recordings provided additional insight into the nature of the stressrelated changes in the BLA. Of particular note, while chronic stress exposure did not alter AMPAR/NMDAR ratio or peak NMDAR-mediated excitatory postsynaptic currents in pyramidal neurons in the BLA, it did significantly increase the frequency of spontaneous excitatory postsynaptic currents in the presence of the $\mathrm{GABA}_{\mathrm{A}}-\mathrm{R}$ antagonist picrotoxin. This demonstrates that stress exposure either led to an increase in glutamate release or an enhancement of pyramidal neuron network activity in the BLA that was revealed only when $\mathrm{GABA}_{\mathrm{A}}-\mathrm{R}$ were blocked. The latter seems more likely given there was no indication of a stress-related increase in spontaneous EPSC amplitude in the presence of the sodium channel blocker tetrodotoxin, which occludes network activity but leaves glutamate release intact. Another key finding was a network-independent (i.e. tetrodotoxin-insensitive) increase in the amplitude of GABAergic miniature inhibitory postsynaptic currents in BLA. Taken together, this pattern of alterations is consistent with an enhancement of network excitability of BLA glutamatergic pyramidal neurons, together with a degree of compensatory upregulation of GABAergic inhibitory transmission. As such, these data add an important line of physiological evidence to support a model in which stress causes restricted increases in BLA network hyperexcitability and resultant alterations in anxietylike behavior. 
As noted above, activation of GluK1 has been shown to increase GABAergic transmission in the BLA. In this context, the finding that intra-BLA infusion of ATPA rescued stressinduced changes in anxiety-like behavior could be explained by a GluK1-mediated increase in GABAergic activity that effectively opposes the network hyperexcitability caused by stress. However, there is also evidence that GluK1 activation can increase presynaptic glutamate release in the rat BLA (Aroniadou-Anderjaska et al., 2012). Furthermore, infusion of ATPA into the rat BLA has been shown to increase anxiety-like behavior in the LDE test (Aroniadou-Anderjaska et al., 2012; Lack et al., 2008) - an effect opposite to that found in the current study and the Wu et al. (2007) study, both using mice. The reason for these discrepancies is unclear and could reflect genuine species differences in the localization and/or function of GluK1 in the BLA. Alternatively, some authors have posited that GluK1 may have varying actions on neurotransmission depending on the concentration of endogenous glutamate in the BLA, with GluK1 effects on GABA predominating under low concentrations, and effects on glutamate dominating at high concentrations (Braga et al., 2003). In this scheme, the effects of ATPA in the current study would have worked against a background of low glutamate. However, this remains speculative in the absence of more direct evidence and these are clearly important questions that remain to be clarified in future work.

The role of other glutamatergic signaling molecules in the stress-induced changes in anxiety behavior we observed also remains to be determined. One potential candidate is GluN1 NMDAR subunit, given prior data indicating an increase in BLA GluN1 mRNA expression after chronic stress (Mozhui et al., 2010). Intra-BLA infusion of AP5 has also previously been shown to reverse anxiogenic-like effects produced by GABA $_{A}-\mathrm{R}$ blockade (Sajdyk and Shekhar, 1997). In the current experiments, infusion of the subunit non-specific NMDAR antagonist AP5 into the BLA of non-stressed mice mimicked the increased light compartment exploration produced by chronic stress. In contrast to the anxio-selective changes produced by stress, however, AP5 produced a profile more consistent with generalized locomotor hyperactivity or behavioral disinhibition - significantly increasing the total distance as well as the distance traveled in both the (aversive) light and (safe) dark compartments of the apparatus. Potent disinhibitory/locomotor hyperactivity effects of systemically administered NMDAR antagonists in mice are well known (e.g., Chen and Holmes, 2009; Karlsson et al., 2008; Shen and Phillips, 1998), and the current data show that these drugs can have a similar effect when locally delivered in the BLA. Unfortunately however, the non-specific behavioral profile produced by AP5 largely prevented meaningful interpretation of the potential modulatory effect of the drug on stress-induced anxiety-related behavior. As such, elucidating the potential contribution of GluN1 to stress-induced anxietylike behavior will require additional studies.

\section{Conclusions}

Providing new insight into glutamatergic alterations in the BLA that underlie changes in anxiety-like behavior due to chronic stress, the current study demonstrated that infusion of the GluK1 receptor agonist, ATPA, normalized stress-induced anxiety-like behavior. A similar experiment involving intra-BLA infusion the GluN1 antagonist AP5 was confounded by profound behavioral disinhibition. Parallel ex vivo physiological analyses of BLA 
synaptic transmission demonstrated a potent GABAergic facilitating effect of ATPA, and revealed evidence of exaggerated glutamatergic pyramidal neuronal network activity following chronic stress. Collectively, these data lead to a model of stress-induced BLA hyperexcitability that is reversed by GluK1-mediated enhancement of GABAergic neurotransmission to normalize anxiety-like behavior. These findings could have implications for understanding the pathophysiology of stress-related anxiety disorders and could suggest novel therapeutic approaches for these disorders that, such GluK1 agonists, target the glutamate system.

\section{Acknowledgments}

We are grateful to Olena Bukalo for critical comments. Research supported by the U.S. Department of Defense Award W81XWH-10-1-0999 to TLK and AH (administered by The Henry M. Jackson Foundation for the Advancement of Military Medicine Inc.), the National Institute on Alcohol Abuse and Alcoholism intramural research program (AH) and the Bowles Center for Alcohol Studies (TLK).

\section{References}

Aroniadou-Anderjaska V, Pidoplichko VI, Figueiredo TH, Almeida-Suhett CP, Prager EM, Braga MF. Presynaptic facilitation of glutamate release in the basolateral amygdala: a mechanism for the anxiogenic and seizurogenic function of GluK1 receptors. Neuroscience. 2012; 221:157-169. [PubMed: 22796081]

Braga MF, Aroniadou-Anderjaska V, Xie J, Li H. Bidirectional modulation of GABA release by presynaptic glutamate receptor 5 kainate receptors in the basolateral amygdala. J Neurosci. 2003; 23 (2):442-452. [PubMed: 12533604]

Caspi A, Hariri AR, Holmes A, Uher R, Moffitt TE. Genetic sensitivity to the environment: the case of the serotonin transporter gene and its implications for studying complex diseases and traits. Am J Psychiatry. 2010; 167 (5):509- 527. [PubMed: 20231323]

Chen YC, Holmes A. Effects of topiramate and other anti-glutamatergic drugs on the acute intoxicating actions of ethanol in mice: modulation by genetic strain and stress. Neuropsychopharmacology. 2009; 34 (6):1454-1466. [PubMed: 18843265]

Christian DT, Alexander NJ, Diaz MR, McCool BA. Thalamic glutamatergic afferents into the rat basolateral amygdala exhibit increased presynaptic glutamate function following withdrawal from chronic intermittent ethanol. Neuropharmacology. 2013; 65:134-142. [PubMed: 22982568]

Crawley JN. Neuropharmacologic specificity of a simple animal model for the behavioral actions of benzodiazepines. Pharmacol Biochem Behav. 1981; 15 (5):695- 699. [PubMed: 6118883]

Cryan, CF.; Dev, KK. The glutamatergic system as a potential therapeutic target for the treatment of anxiety disorders. Blanchard, RJ.; Blanchard, DC.; Nutt, DJ., editors. 2007.

Duvarci S, Pare D. Glucocorticoids enhance the excitability of principal basolateral amygdala neurons. J Neurosci. 2007; 27 (16):4482-4491. [PubMed: 17442833]

Griebel G, Holmes A. Fifty years of hurdles and hope in anxioylitc drug discovery. Nat Rev Drug Discov. 2013; 12 (9):667-687. [PubMed: 23989795]

Gunduz-Cinar O, Macpherson KP, Cinar R, Gamble-George J, Sugden K, Williams B, et al. Convergent translational evidence of a role for anan-damide in amygdala-mediated fear extinction, threat processing and stress-reactivity. Mol Psychiatry. 2013; 18 (7):813-823. [PubMed: 22688188]

Holmes A, Fitzgerald PJ, Macpherson KP, Debrouse L, Colacicco G, Flynn SM, et al. Chronic alcohol remodels prefrontal neurons and disrupts NMDAR-mediated fear extinction encoding. Nat Neurosci. 2012; 15 (10):1359-1361. [PubMed: 22941108]

Holmes A, Parmigiani S, Ferrari PF, Palanza P, Rodgers RJ. Behavioral profile of wild mice in the elevated plus-maze test for anxiety. Physiol Behav. 2000; 71 (5):509-516. [PubMed: 11239669]

Holmes A, Singewald N. Individual differences in recovery from traumatic fear. Trends Neurosci. 2013; 36 (1):23-31. [PubMed: 23260015] 
Humeau Y, Reisel D, Johnson AW, Borchardt T, Jensen V, Gebhardt C, et al. A pathway-specific function for different AMPA receptor subunits in amygdala long-term potentiation and fear conditioning. J Neurosci. 2007; 27 (41):10947-10956. [PubMed: 17928436]

Ihne JL, Fitzgerald PJ, Hefner KR, Holmes A. Pharmacological modulation of stress-induced behavioral changes in the light/dark exploration test in male C57BL/6J mice. Neuropharmacology. 2012; 62 (1):464-473. [PubMed: 21906605]

Karlsson RM, Tanaka K, Heilig M, Holmes A. Loss of glial glutamate and aspartate transporter (excitatory amino acid transporter 1) causes locomotor hyperactivity and exaggerated responses to psychotomimetics: rescue by haloperidol and metabotropic glutamate $2 / 3$ agonist. Biol Psychiatry. 2008; 64 (9):810-814. [PubMed: 18550032]

Kavushansky A, Richter-Levin G. Effects of stress and corticosterone on activity and plasticity in the amygdala. J Neurosci Res. 2006; 84 (7):1580-1587. [PubMed: 16998919]

Kim SY, Adhikari A, Lee SY, Marshel JH, Kim CK, Mallory CS, et al. Diverging neural pathways assemble a behavioural state from separable features in anxiety. Nature. 2013; 496 (7444):219223. [PubMed: 23515158]

Krishnan V, Han MH, Graham DL, Berton O, Renthal W, Russo SJ, et al. Molecular adaptations underlying susceptibility and resistance to social defeat in brain reward regions. Cell. 2007; 131 (2):391-404. [PubMed: 17956738]

Lack AK, Ariwodola OJ, Chappell AM, Weiner JL, McCool BA. Ethanol inhibition of kainate receptor-mediated excitatory neurotransmission in the rat basolateral nucleus of the amygdala. Neuropharmacology. 2008; 55 (5):661-668. [PubMed: 18617194]

Li C, Pleil KE, Stamatakis AM, Busan S, Vong L, Lowell BB, et al. Pre-synaptic inhibition of gammaaminobutyric acid release in the bed nucleus of the stria terminalis by kappa opioid receptor signaling. Biol Psychiatry. 2012; 71 (8):725-732. [PubMed: 22225848]

Li H, Chen A, Xing G, Wei ML, Rogawski MA. Kainate receptor-mediated heterosynaptic facilitation in the amygdala. Nat Neurosci. 2001; 4 (6):612-620. [PubMed: 11369942]

Li H, Rogawski MA. GluR5 kainate receptor mediated synaptic transmission in rat basolateral amygdala in vitro. Neuropharmacology. 1998; 37 (10-11):1279-1286. [PubMed: 9849665]

Maroun M, Ioannides PJ, Bergman KL, Kavushansky A, Holmes A, Wellman CL. Fear extinction deficits following acute stress associate with increased spine density and dendritic retraction in basolateral amygdala neurons. Eur J Neurosci. 2013; 38 (4):2611-2620. [PubMed: 23714419]

Mozhui K, Karlsson RM, Kash TL, Ihne J, Norcross M, Patel S, et al. Strain differences in stress responsivity are associated with divergent amygdala gene expression and glutamate-mediated neuronal excitability. J Neurosci. 2010; 30 (15):5357-5367. [PubMed: 20392957]

Nagura H, Ishikawa Y, Kobayashi K, Takao K, Tanaka T, Nishikawa K, et al. Impaired synaptic clustering of postsynaptic density proteins and altered signal transmission in hippocampal neurons, and disrupted learning behavior in PDZ1 and PDZ2 ligand binding-deficient PSD-95 knockin mice. Mol Brain. 2012; 5:43. [PubMed: 23268962]

Reznikov LR, Reagan LP, Fadel JR. Effects of acute and repeated restraint stress on GABA efflux in the rat basolateral and central amygdala. Brain Res. 2009; 1256:61-68. [PubMed: 19124010]

Roozendaal B, McEwen BS, Chattarji S. Stress, memory and the amygdala. Nat Rev Neurosci. 2009; 10 (6):423-433. [PubMed: 19469026]

Sajdyk TJ, Shekhar A. Excitatory amino acid receptor antagonists block the cardiovascular and anxiety responses elicited by gamma-aminobutyric acid A receptor blockade in the basolateral amygdala of rats. J Pharmacol Exp Ther. 1997; 283 (2):969-977. [PubMed: 9411030]

Shen EH, Phillips TJ. MK-801 potentiates ethanol's effects on locomotor activity in mice. Pharmacol Biochem Behav. 1998; 59 (1):135-143. [PubMed: 9443548]

Singewald N. Altered brain activity processing in high-anxiety rodents revealed by challenge paradigms and functional mapping. Neurosci Biobehav Rev. 2007; 31 (1):18-40. [PubMed: 16620984]

Surget A, Wang Y, Leman S, Ibarguen-Vargas Y, Edgar N, Griebel G, et al. Corticolimbic transcriptome changes are state-dependent and region-specific in a rodent model of depression and of antidepressant reversal. Neuropsychopharmacology. 2009; 34 (6):1363-1380. [PubMed: 18536703] 
Uchida S, Hara K, Kobayashi A, Otsuki K, Yamagata H, Hobara T, et al. Epigenetic status of Gdnf in the ventral striatum determines susceptibility and adaptation to daily stressful events. Neuron. 2011; 69 (2):359-372. [PubMed: 21262472]

Walker DL, Ressler KJ, Lu KT, Davis M. Facilitation of conditioned fear extinction by systemic administration or intra-amygdala infusions of D-cyclo-serine as assessed with fear-potentiated startle in rats. J Neurosci. 2002; 22 (6):2343-2351. [PubMed: 11896173]

Whittle N, Hauschild M, Lubec G, Holmes A, Singewald N. Rescue of impaired fear extinction and normalization of cortico-amygdala circuit dysfunction in a genetic mouse model by dietary zinc restriction. J Neurosci. 2010; 30 (41):13586-13596. [PubMed: 20943900]

Wu LJ, Ko SW, Toyoda H, Zhao MG, Xu H, Vadakkan KI, et al. Increased anxiety-like behavior and enhanced synaptic efficacy in the amygdala of GluR5 knockout mice. PLoS One. 2007; 2 (1):e167. [PubMed: 17245443]

Yang RJ, Mozhui K, Karlsson RM, Cameron HA, Williams RW, Holmes A. Variation in mouse basolateral amygdala volume is associated with differences in stress reactivity and fear learning. Neuropsychopharmacology. 2008; 33 (11):2595-2604. [PubMed: 18185497]

\section{Further reading}

Camp M, Norcross M, Whittle N, Feyder M, D’Hanis W, Yilmazer-Hanke D, et al. Impaired Pavlovian fear extinction is a common phenotype across genetic lineages of the 129 inbred mouse strain. Genes Brain Behav. 2009; 8 (8):744- 752. [PubMed: 19674120]

Dobrunz LE, Stevens CF. Heterogeneity of release probability, facilitation, and depletion at central synapses. Neuron. 1997; 18 (6):995-1008. [PubMed: 9208866]

Farley S, Dumas S, El Mestikawy S, Giros B. Increased expression of the Vesicular Glutamate Transporter-1 (VGLUT1) in the prefrontal cortex correlates with differential vulnerability to chronic stress in various mouse strains: effects of fluoxetine and MK-801. Neuropharmacology. 2012; 62 (1):503-517. [PubMed: 21945287]

Ibarguen-Vargas Y, Surget A, Touma C, Palme R, Belzung C. Multifaceted strain-specific effects in a mouse model of depression and of antidepressant reversal. Psychoneuroendocrinology. 2008; 33 (10):1357-1368. [PubMed: 18790573]

Jacobson LH, Cryan JF. Feeling strained? Influence of genetic background on depression-related behavior in mice: a review. Behav Genet. 2007; 37 (1):171-213. [PubMed: 17029009]

Kash TL, Baucum AJ 2nd, Conrad KL, Colbran RJ, Winder DG. Alcohol exposure alters NMDAR function in the bed nucleus of the stria terminalis. Neuropsychopharmacology. 2009; 34 (11): 2420-2429. [PubMed: 19553918]

Kullmann DM, Erdemli G, Asztely F. LTP of AMPA and NMDA receptor-mediated signals: evidence for presynaptic expression and extrasynaptic glutamate spill-over. Neuron. 1996; 17 (3):461-474. [PubMed: 8816709]

Millstein RA, Ralph RJ, Yang RJ, Holmes A. Effects of repeated maternal separation on prepulse inhibition of startle across inbred mouse strains. Genes Brain Behav. 2006; 5 (4):346-354. [PubMed: 16716204]

\section{Appendix A. Supplementary data}

Supplementary data related to this article can be found at http://dx.doi.org/10.1016/ j.neuropharm.2014.04.015. 

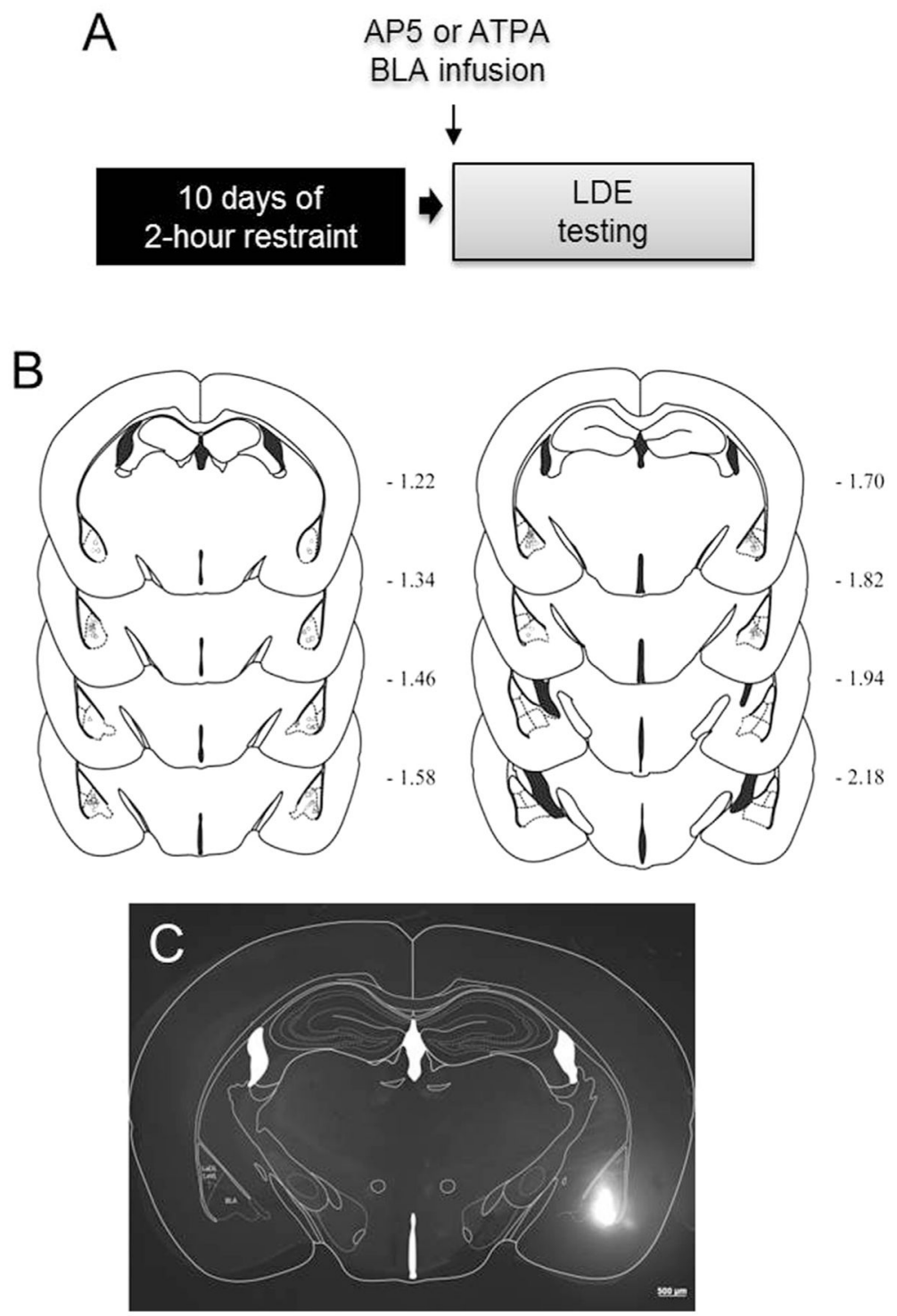

Fig. 1. Testing effects of intra-BLA infusion of glutamate drugs on stress-induced anxiety-like behavior

(A) Schematic of experimental design to test for the effects of intra basolateral amygdala (BLA) infusion of the GluN1 antagonist AP5 or the GluK1 agonist ATPA on behavior in the light/dark exploration test (LDE) following chronic restraint stress. (B) Coronal sections indicating site of cannula placements in BLA. (C) Epifluorescent image of a fluorophore infused into BLA estimating location and spread of infused drugs. 

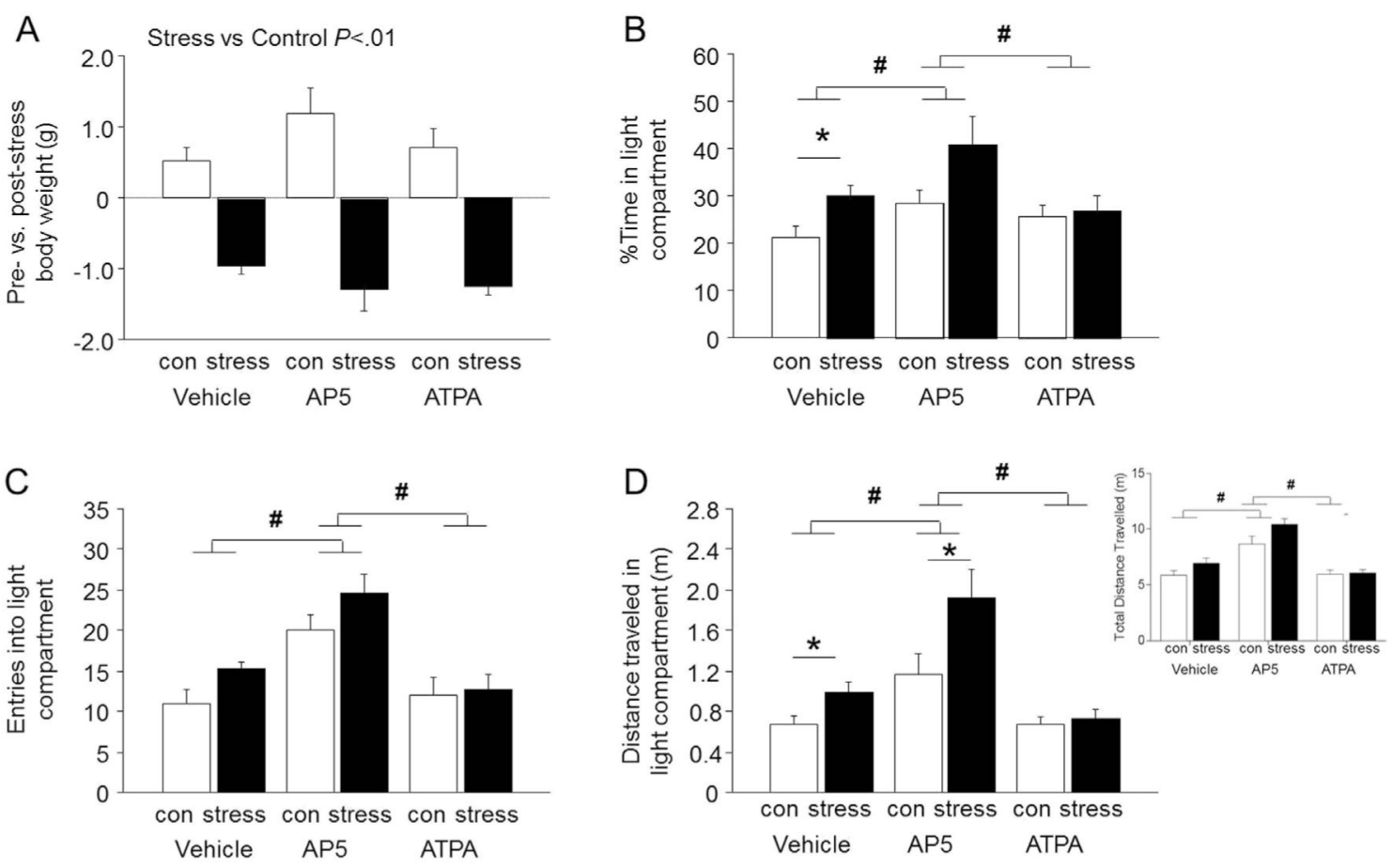

Fig. 2. Effects of intra-BLA infusion of AP5 or ATPA on stress-induced anxiety-like behavior (A) Stressed mice showed significantly reduced body weight as compared to non-stressed controls (con). (B) Stressed mice spent a greater percentage of time in the light compartment than non-stressed controls following intra-BLA infusion of vehicle, but not after intra-BLA AP5 or ATPA infusion. Mice infused with AP5 showed spent more time in the light compartment than vehicle-infused mice, irrespective of stress. (C) Mice infused with AP5 made more entries into the light compartment than vehicle-infused controls, irrespective of stress. (D) Stressed mice traveled farther overall (inset) as well as in the light compartment than non-stressed controls following infusion of vehicle, but not after intra-BLA ATPA infusion. Mice infused with AP5 traveled farther overall and in the light dark compartment than vehicle-infused controls, an effect which was augmented by stress. Data are means \pm SEM. $* P<.05$ stress versus non-stressed controls receiving the same treatment, $\# P<.05$ AP5 versus vehicle or ATPA. $n=6-9$ per group. 

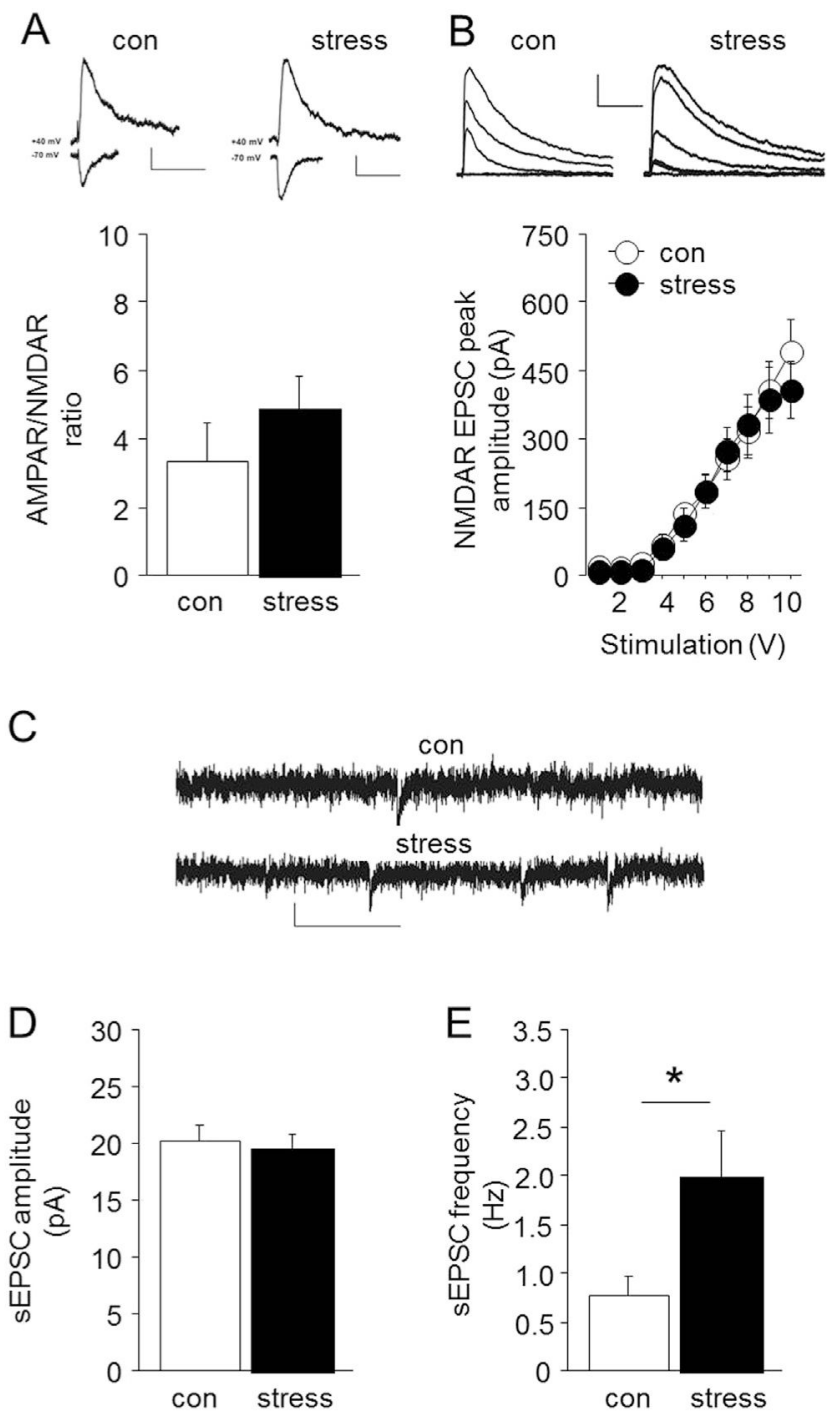

Fig. 3. Effects of stress on BLA synaptic transmission (A) Stressed mice showed similar AMPAR/NMDAR ratio as non-stressed controls. Representative traces show AMPAR/NMDAR ratio in non-stressed controls (con) and stressed mice (scale bars $=10 \mathrm{pA}, 50 \mathrm{~ms}$ for ratio). (B) Stressed mice showed similar electrically-evoked NMDAR EPSC peak amplitude as non-stressed controls. Representative traces show EPSCs recorded from non-stressed controls and stressed mice (scale bars $=20$ pA, 200 ms). (C) Representative traces show spontaneous EPSCs recorded from nonstressed controls (con) and stressed mice (scale bars $=200$ pA, $100 \mathrm{~ms}$ ). Stressed mice showed normal spontaneous EPSC amplitude (D) but significantly increased spontaneous EPSC frequency $(\mathbf{E})$, as compared to non-stressed controls. Data are means \pm SEM. $* P<$. 05 stress versus non-stressed controls. $n=8-9$ per group. 
A

sEPSCs
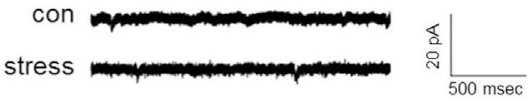

B

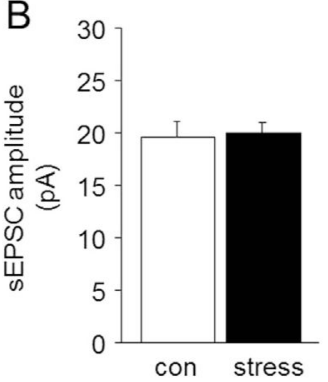

C

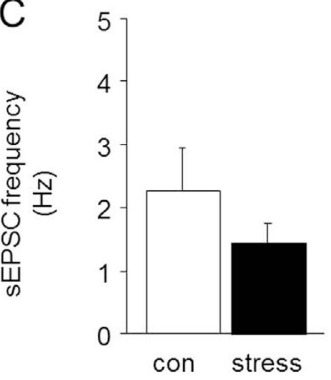

G

$$
\text { mEPSCs }
$$
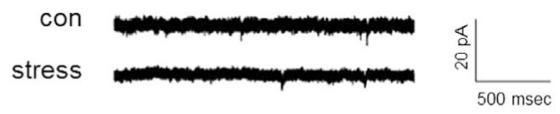
stressed controls. $n=6-7$ per group.
D
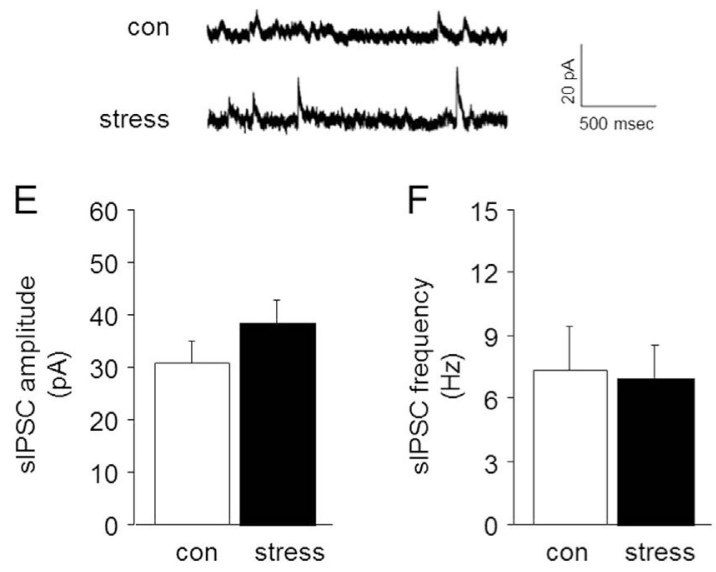

$J$
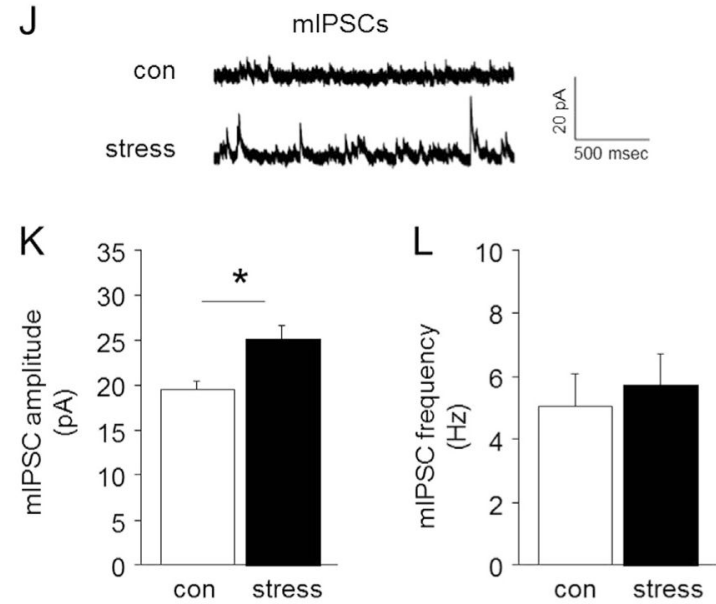

Fig. 4. Effects of stress on BLA spontaneous and miniature synaptic transmission

(A) Representative traces for spontaneous EPSCs. Stressed mice showed a similar amplitude

(B) and frequency (C) of spontaneous EPSCs, as compared to non-stressed controls (con).

(D) Representative traces for spontaneous IPSCs. The amplitude (E) and frequency (F) of spontaneous IPSCs was similar between stressed and non-stressed mice. (G) Representative traces for miniature EPSCs. Stressed mice showed a similar amplitude $(\mathbf{H})$ and frequency $(\mathbf{I})$ of miniature EPSCs, relative to non-stressed controls. (J) Representative traces for miniature IPSCs. Stressed mice showed increased miniature IPSC amplitude $(\mathbf{K})$, but not frequency $(\mathbf{L})$, relative to non-stressed controls. Data are means \pm SEM. $* P<.05$ stress versus non- 